\title{
PENSAMIENTO EDUCATIVO IMPLÍCITO EN LAS PRÁCTICAS DE ENSEÑANZA DE LA BIOLOGÍA
}

—Un acercamiento a su explicitación a través de la investigación en el aula—

\author{
Chona D. Guillermo, Arteta de Molina Judith E., Martínez de Rueda Sonia*1 \\ Departamento de Biología
}

\begin{abstract}
Biology educators' beliefs about teaching are very important. This qualitative investigation pretended to establish the influence of the belief system upon teacher's actions. Biology teacher's reflections about their own actions can contribute to the transformation of their pedagogic practice. The purpose of this article is to present and discuss some research outcomes respect to aspects that affect his/her practice like pedagogic, didactic, learning, conceptions about biologic knowledge, and teacher's image, therefore them influence.
\end{abstract}

\section{RESUMEN}

Las creencias de Los profesores acerca de la enseñanza son muy importantes. Esta investigación cualitativa pretendió establecer la influencia del sistema de creencias que sustenta las acciones del docente. Las reflexiones de los profesores de Biología sobre sus propias acciones pueden contribuir a la transformación de su práctica pedagógica. El propósito de este artículo es presentar y discutir algunos hallazgos investigativos con relación a aspectos que afectan su práctica como los pedagógicos, didácticos, aprendizaje, concepciones sobre el conocimiento biológico y la imagen de la profesión, así como sus influencias.

\section{INTRODUCCIÓN}

El estudio sobre la actuación del profesor en el aula y su relación con el pensamiento que dirige su acción educativa, cobra particular importancia hoy cuando se cuestiona al docente respecto de su papel en la sociedad y se le exige educar con calidad a jóvenes en un mundo globalizado. El pensamiento del docente de Biología acerca de la ciencia y los aspectos educativos se vislumbra en a forma como organiza y desarrolla su trabajo, las interacciones que posibilita con sus estudiantes y las razones en las que sustenta las múltiples orientaciones y criterios involucrados en su trabajo como docente.

La participación del docente en la identificación y reflexión sobre la realidad de su quehacer y el conocimiento de los referentos de sus acciones, favorece las transformaciones conscientes de su práctica pedagógica, en la perspectiva de dar sentido a las acciones respecto de la formación deseada en sus estudiantes; de igual manera se contribuye a mejorar a calidad de la enseñanza de las ciencias y se posibilita a consolidación de una comunidad académica de docentes investigadores.

\footnotetext{
${ }^{1}$ Profesores Unfversidad Pedagógica Nacional
} 


\section{REFERENTES}

\section{PENSAMIENTO DEL PROFESOR}

Para abordar el estudio sobre el pensamiento implícito en las acciones del docente, se cuenta con una buena base de trabajos y de referentes conceptuales, que han surgido de los distintos debates donde el centro de la mirada es el profesor. desarrollándose un enfoque particular sobre el pensamiento del profesor. A propósito de este interés, Marcelo (1987) propone coleccionar, cotear e interpretar el conocimiento práctico del profesor. Gallego (1991), hace una revisión del desarrollo de este tema y destaca que el profesor posee pensamientos que mediatiza con la acción, la cual está directamente influenciada por la imagen que posee de su profesión. La autora resalta conceptos y aproximaciones como: las creencias, el conocimiento práctico, las perspectivas, los criterios de enseñanza subjetivos, las imágenes, las teorías de acción del profesor; formalizando con todas éstas, un criterio denominado conocimiento profesional del profesor".

Son, entonces varios los autores cuyo centro del trabajo investigativo ha sido el pensamiento de Los profesores y sus creencias, tal es el caso de Barquín (1987) y Gallego (1991), quienes plantean que el conocimiento y las creencias, constituyen, en el profesor, su saber pedagógico, el cual incide en su comportamiento como profesional, en la vida de la escuela y en la de los estudiantes.

Pérez y Gimeno (1988), muestran que existen dos enfoques principales en a investigación sobre el pensamiento del profesor, una, eminentemente psicologista preocupada por discriminar los procesos formales de procesamiento de la información y otra pedagógica, centrada en los contenidos, ideas y teorías del profesor sobre los procesos de enseñanza y aprendizaje. Siguiendo este último enfoque, ellos consideran que el conocimiento del profesor es "tácito, poco articulado y organizado con una lógica peculiar, que responde más a las prioridades afectivas establecidas en la historia personal - profesional', que a los requerimientos de las teorías pedagógicas.

\section{CREENCIAS}

Las creencias influyen en la forma en que se caracterizan los acontecimientos y en cómo estimamos la variación simultánea de fenómenos. Incluso la contrastación de nuestras creencias con la experiencia, está influida por las propias creencias y por su interacción con la manera cómo percibimos los datos (Munby, 1982); de ahí que como bien lo destaca Furió (1994), el profesor es un factor clave que determina en gran medida el éxito o el fracaso de cualquier innovación curricular o la implementación de un modelo educativo. Ahora bien, los profesores, sobretodo con más experiencia, tienen normas y principios de actuación en ja práctica que guían su planificación y su conducta interactiva (Reyes y Salcedo, 1998).

De igual forma, otros autores ponen en evidencia que las creencias de los profesores de ciencias acerca de su práctica y del aprendizaje de los estudiantes están más relacionados con su sistema de concepciones, que con los mandatos de los nuevos currículos. (Yerrit et. al. 1997).

Otros planteamientos que facilitan las distintas interpretaciones en este tipo de trabajos, como los de Porlán et al. (1997), muestran los argumentos teóricos que fundamentan la investigación en la escuela y que favorecen tanto la evolución como el desarrollo de los 
estudiantes y de los profesores, resumidos en tres grandes perspectivas: La perspectiva constructivista, la perspectiva sistémica y compleja y la perspectiva crítica.

En Chona et al. (1998), se recogen diversas expresiones de investigadores que han conceptualizado lo que puede considerarse creencia (Rockeach, 1968; Dewey, 1989; Gallego, 1991). Además, Mellado (1996) retoma la discusión no sólo sobre la conceptualización del término, sino también sobre las implicaciones que puedan tener los distintos tratamientos de la problemática acerca de creencias o concepciones en los profesores.

Este horizonte conceptual fundamenté en parte, las búsquedas de sentido para nuestro trabajo en la interpretación de la práctica de los profesores de Biología y su relación con sus creencias.

\section{CONTEXTO DE LA INVESTIGACIÓN}

Durante los últimos cinco años hemos adelantado investigaciones tratando de caracterizar el pensamiento de algunos docentes de Biología en Santa Fe de Bogotá, específicamente, sobre concepciones relacionadas con pedagogía, aprendizaje, didáctica, biología e imagen de la profesión docente (Chona, et al., 1998). Los resultados muestran la existencia de creencias fuertemente arraigadas en el pensamiento del profesor, que se mantienen a pesar de todos los cambios y flexibilidad que introduce la nueva normatividad educativa nacional.

El proyecto de investigación acerca de "Estudio de la práctica del profesor de Biología y su relación con sus creencias", que los autores desarrollamos y que origina este escrito, pretendió determinar en profesores de básica secundaria, la presencia de algunas creencias sobre los aspectos señalados arriba y analizar de qué maneras estas creencias influían en la actuación del profesor.

En esta investigación, reconocemos al maestro como eje fundamental en el proceso educativo y sin cuya decisiva y consciente participación no es posible consolidar las propuestas de innovación. Por ello es urgente determinar las condiciones que le impiden al maestro realmente transformar su práctica, puesto que muchos estudios en Didáctica y en Enseñanza de las ciencias están centrados en problemas relativos al alumno, a la enseñanza y al aprendizaje (Giordan, 1982; Gaglardí, 1986; Novak y Gowin, 1988). Pero a pesar de todos estos trabajos, no se ha logrado producir una modificación significativa en la enseñanza y en la actuación del profesor (Pacca et al. 1997).

Esto nos llevó a hacer un seguimiento a la acción directa del profesor en el aula, en la idea de contrastar e interpretar en la actuación del docente, el verdadero sentido de su práctica pedagógica y proponer algunos análisis del pensamiento del profesor de Biología, haciendo explicitas las relaciones con su práctica. La problemática expuesta se constituyó en el objeto de este trabajo, lo cual permitió ofrecer algunos criterios para que los maestros caracterizaran y cuestionaran su propia actuación y generaran imágenes de cambio acordes con las características de su propio contexto de actuación.

Para nuestro estudio asumimos, inicialmente, como creencias las expresiones verbales o escritas que muestran parte del pensamiento del profesor y que se reflejan en su acción docente; "la creencia.., abarca todas las cuestiones sobre las cuales no disponemos de un conocimiento seguro pero en las que confiamos lo suficiente como para actuar de acuerdo 
con ellas, y también cuestiones que ahora aceptamos como indudablemente verdaderas, como conocimiento, pero que pueden ser cuestionadas en el futuro..." (Dewey, 1989). Al referirnos a creencia nosotros no estamos considerando aspectos del pensamiento mitico o religioso del profesor, sino las ideas relacionadas con pedagogía, didáctica, aprendizaje, conocimiento biológico e imagen de la profesión docente: que se constituyeron en nuestras categorías de análisis.

\section{ENFOQUE METODOLÓGICO}

El presente estudio se realizó desde una metodología de investigación cualitativa, con un enfoque etnográfico. El abordaje metodológico de este estudio implicó el análisis descriptivo del contexto y la interpretación.

El análisis descriptivo se dio a través de participación en el contexto específico y registro de lo que allí sucedía, mediante la realización de notas de campo, encuestas, grabaciones, filmaciones, entrevistas y recopilación de documentos. Se adelantó la reflexión analítica del registro documental con miras a identificar las creencias en cada maestro y contrastarlas con las características de su práctica. Todo ello condujo a una descripción detallada del registro obtenido, contrastando las diferentes fuentes de información.

La interpretación implicó el trabajo conjunto de investigadores y profesores, mediante la realización de talleres con los profesores participantes en la investigación en los cuales se realizó la reflexión sobre el análisis descriptivo, para construcción conjunta de sentido. El trabajo de investigación se desarrolló con seis docentes del Distrito Capital, en el nivel de la básica secundaria que se desempeñan en el área de Ciencias Naturales y Educación Ambiental, con énfasis en aspectos Biológicos.

El análisis de las acciones y expresiones de los docentes, nos permitieron vislumbrar elementos enunciativos de sus creencias que contrastamos e interpretamos con el docente en el aula, para empezar a explicitar su pensamiento pedagógico y relacionarlo con su práctica. La problemática expuesta se constituyó en el centro de este trabajo, lo cual permitió ofrecer algunos criterios para que los maestros cuestionaran su propia acción y generaran procesos de transformación educativa acordes con las nuevas visiones elaboradas.

\section{RESULTADOS}

\section{Pensamiento y acciones del profesor. Algunas relaciones}

La información obtenida con respecto a cada docente (cuyo nombre ha sido cambiado para proteger su identidad), se analizo e interpreto con los participantes, como un caso de estudio. A partir de ello fue posible detectar algunas de las creencias a las cuales hacíamos seguimiento (ver Anexo 1); pero además, fue interesante y complejo el hecho que, al querer dar significado y construir sentido a los hallazgos encontrados e intentando develar si existía o no una lógica en la actuación y pensamiento del profesor, se explicitaron ciertas relaciones y particularidades que aparecen en cada caso estudiado. Pero ¿qué sentido le podríamos asignar a estos hallazgos?, ¿que tienen que ver con las realidades de la actuación del docente? Estamos convencidos que las caracterizaciones realizadas, de alguna manera reflejan el pensamiento educativo de los profesores; esta 
afirmación se apoya en Cohen y Manion (1990), quienes señalan que dada la complejidad de las situaciones abordadas en el estudio de casos, resulta conveniente hacer triangulación, que para el caso de esta investigación se realizó para tiempos, espacios, investigadores y fuentes de información, complementado todo ello, con la devolución y revisión de la información con los maestros participantes.

El resumen elaborado para cada caso, (ver Cuadro No. 1), permite recoger y precisar elementos en el pensamiento y acción del profesor, en torno a las categorías que se han utilizado en este estudio. Cabe destacar, la diversidad de puntos de vista en los maestros (ver por ejemplo, concepción de aprendizaje o de educación, al leer horizontalmente los distintos casos), en contraste con la unidad que se encuentra al leer cada caso de manera vertical (véase caña caso en particular).

Por otra parte, en este cuadro se pueden ver también que algunos aspectos o parámetros, a nuestro modo de ver, se constituyen en ideas muy arraigadas en el pensamiento del profesor y que posiblemente sirven de referente o anclaje a otras creencias que pudiesen ser mas explícitas en la actuación del docente. Es así como, revisando la caracterización del profesor y sus clases, en contraste con las creencias detectadas, encontramos algunas relaciones que nos hacen suponer la subordinación de unas creencias a otras, dentro del esquema de pensamiento del profesor.

En nuestro estudio se evidencia, que la concepción de educación y la concepción de ciencia que tenga un docente, determina en gran medida, lo que el profesor posibilite respecto al tipo de orientación en las relaciones profesor-alumno, alumno-alumno, alumno-saber, profesor-saber, alumno-realidad social, profesor-profesor, entre otras. De igual manera, la concepción del profesor sobre el saber que enseña, en este caso la Biología, se relaciona con el papel asignado al texto en el aula, al uso del laboratorio o a las salidas al campo.

Teniendo en cuenta el análisis de los casos trabajados, la investigación muestra que se presenta bastante coherencia entre las concepciones del profesor y su acción educativa. Es así como, aún cuando las metodologías de enseñanza de un docente. muestren distintas modalidades durante la interacción en el aula, es posible vislumbrar ciertas pautas fijas en las intencionalidades que guían la acción del maestro, que estructuran su pensamiento pedagógico, en el cual influye su formación inicial y su saber práctico. Cabe resaltar que contrario a lo que plantea Mellado (1996), hemos encontrado así, coherencia entre las concepciones del profesor y su actuación en el aula. 
Cuadro No. 1. Caracterización de los profesores.

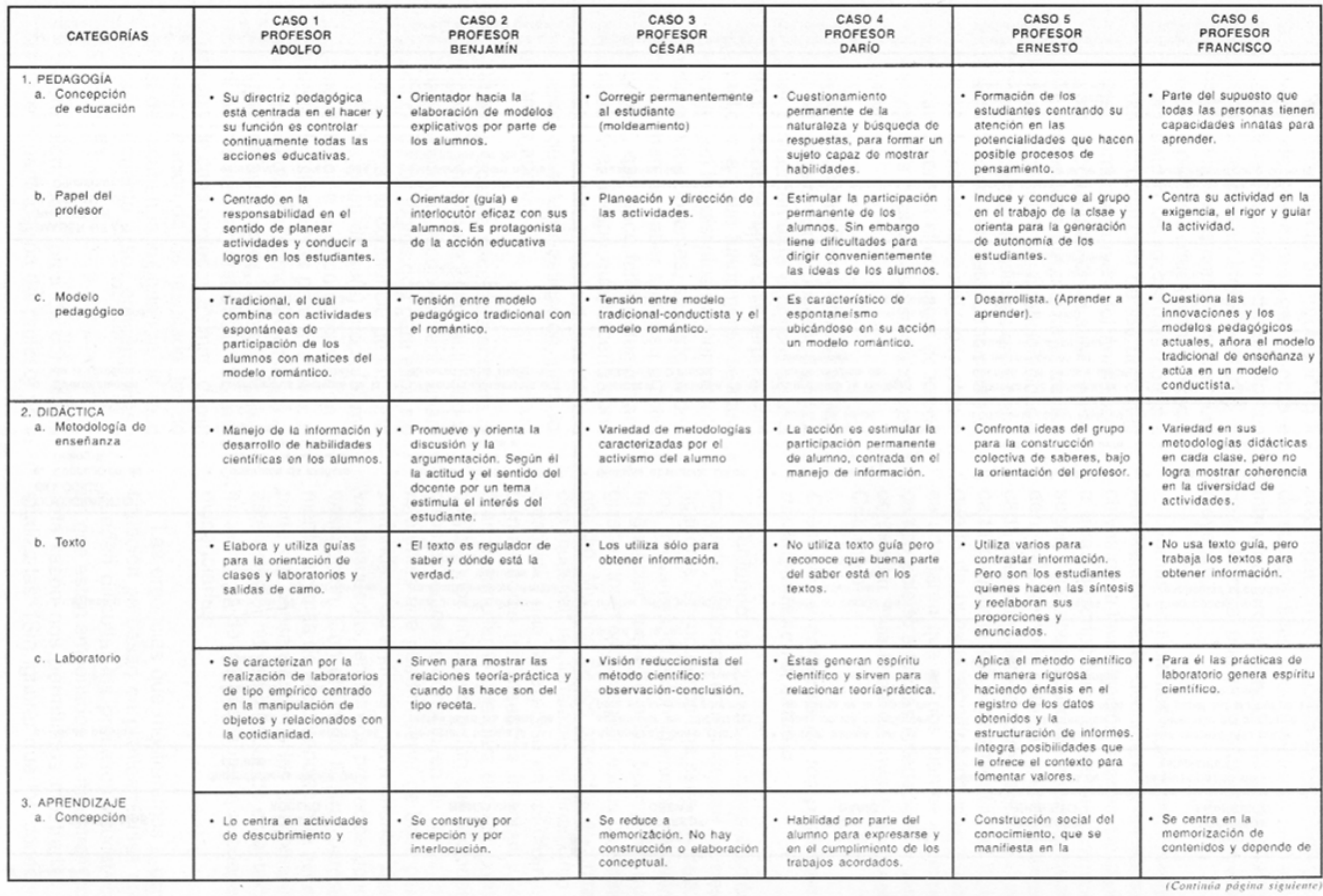




\begin{tabular}{|c|c|c|c|c|c|c|}
\hline CATEGORIAS & $\begin{array}{l}\text { CASO } 1 \\
\text { PROFESOR } \\
\text { ADOLFO }\end{array}$ & $\begin{array}{l}\text { CASO 2 } \\
\text { PROFESOR } \\
\text { BENJAMIN }\end{array}$ & $\begin{array}{l}\text { CASO } 3 \\
\text { PROFESOR } \\
\text { CEESAR }\end{array}$ & $\begin{array}{l}\text { CASO } 4 \\
\text { PROFESOR } \\
\text { DARIO }\end{array}$ & $\begin{array}{l}\text { CASO } 5 \\
\text { PROFESOR } \\
\text { ERNESTO }\end{array}$ & $\begin{array}{l}\text { CASO } 6 \\
\text { PROFESOR } \\
\text { FRANCISCO }\end{array}$ \\
\hline & $\begin{array}{l}\text { depende de la acción del } \\
\text { profesor. }\end{array}$ & & & & $\begin{array}{l}\text { elaboración conjunta de } \\
\text { conceptualizaciones. }\end{array}$ & $\begin{array}{l}\text { la participacion del } \\
\text { estudianto. }\end{array}$ \\
\hline b. Ideas previas & $\begin{array}{l}\text { - Si bion tiene on cuenta las } \\
\text { opiniones de los alumnos } \\
\text { no logra manejar de } \\
\text { manera adocuada las } \\
\text { ideas previas de los } \\
\text { ostudiantos. }\end{array}$ & $\begin{array}{l}\text { - Respeta y trabaja la } \\
\text { profundididad las ideas de } \\
\text { los estudiante. }\end{array}$ & $\begin{array}{l}\text { - Aprendizajo como reto y } \\
\text { superación de dificultades } \\
\text { para surgir como persona. } \\
\text { (No cognitivas). No toma. } \\
\text { en cuenta ideas previas. } \\
\text { Poco se analizan las } \\
\text { inquietudes del estudiante } \\
\text { sobre el tema. }\end{array}$ & $\begin{array}{l}\text { - Si bien trabaja con las } \\
\text { ideas de los estudiantes. } \\
\text { se aparta de la lógica de } \\
\text { ostas y de las rospuostas } \\
\text { por seguir el desarrollo de } \\
\text { la guía. }\end{array}$ & $\begin{array}{l}\text { - Tiere en cuenta las ideas } \\
\text { do los ostudiantes, los } \\
\text { trabaja y discute con todo } \\
\text { of grupo. Realiza } \\
\text { diagnostico inicial antos } \\
\text { de empezar cada curso y } \\
\text { ubica las dificultados do } \\
\text { los niños. }\end{array}$ & $\begin{array}{l}\text { - So intorosa más por el } \\
\text { desatrollo del programa y } \\
\text { ef papool dol alumna es ser } \\
\text { teceptor de ideas. }\end{array}$ \\
\hline c. Evaluación & $\begin{array}{l}\text { - Tieno interós en la } \\
\text { evaluación de actividades } \\
\text { y habilidades. }\end{array}$ & $\begin{array}{l}\text { - Cuestiona las pruebas } \\
\text { tradicionalos poro evalúa } \\
\text { contenido, actifudes y } \\
\text { nabilidados } \\
\text { argumentativas. }\end{array}$ & - Interós en la promoción. & $\begin{array}{l}\text { - Tiene en cuenta las } \\
\text { habilidades para } \\
\text { cuostionar y ser } \\
\text { responsable en los } \\
\text { tabajos on que } \\
\text { compromete al alumno. }\end{array}$ & $\begin{array}{l}\text { - Todas las actividades } \\
\text { intentan favorocer of } \\
\text { aprendizaje s significativo } \\
\text { en los estudiantos. } \\
\text { Evalüa procesos. }\end{array}$ & $\begin{array}{l}\text { - Preocupación por } \\
\text { respuestas acertadas } \\
\text { según el contenido. }\end{array}$ \\
\hline $\begin{array}{l}\text { 1. CONOCIMIENTO } \\
\text { BIOLOGICO } \\
\text { a. Concepción de } \\
\text { Biologia }\end{array}$ & $\begin{array}{l}\text { - Construida de manera } \\
\text { impirica. Rolaciona ol } \\
\text { discurso cientifico con el } \\
\text { roligioso. }\end{array}$ & $\begin{array}{l}\text { - Biologia compioja y dificil } \\
\text { de aprender. La atorda de } \\
\text { manora analitica y } \\
\text { contemplativa. }\end{array}$ & $\begin{array}{l}\text { - Biologia abstracta. Dificil } \\
\text { do trabajar, poro tacil do } \\
\text { aprender. }\end{array}$ & $\begin{array}{l}\text { - Tiene una imagon } \\
\text { empirista de la Biologia y } \\
\text { se construye facilimente si } \\
\text { so progunta y experimenta } \\
\text { sobre los seres. }\end{array}$ & $\begin{array}{l}\text { - Biologia os tácil do } \\
\text { aprender, debe ser } \\
\text { asumida con sontido para } \\
\text { la vida del sujeto y } \\
\text { conoctada con la roalidad. }\end{array}$ & $\begin{array}{l}\text { - Imagon empirista de la } \\
\text { Biologia, pero no fuo } \\
\text { formada en esta disciplina. }\end{array}$ \\
\hline $\begin{array}{l}\text { b. Diferenciación } \\
\text { de ta Biologia }\end{array}$ & $\begin{array}{l}\text { - Dírerencia ia Biologia de is } \\
\text { Fisica y de la Quimica. }\end{array}$ & $\begin{array}{l}\text { - Diferencia claramonte el } \\
\text { ciscurso de la Biologia. }\end{array}$ & $\begin{array}{l}\text { - Direrencia la Biología de la } \\
\text { Fisica y la Quimica. }\end{array}$ & $\begin{array}{l}\text { - Considera la Biologia } \\
\text { como mezica do } \\
\text { disciplinas. }\end{array}$ & $\begin{array}{l}\text { - Diterencis la Biologia, la } \\
\text { quimica y la tisica y ubica } \\
\text { su pertinencis an un } \\
\text { trabajo intordiscipinario. }\end{array}$ & - Sin intormación. \\
\hline c. Saber biologico & $\begin{array}{l}\text { - Se obtiene con la } \\
\text { aplicación del método } \\
\text { cientifico. }\end{array}$ & $\begin{array}{l}\text { - Se obtiene a travós dol } \\
\text { manojo de información y } \\
\text { sensibilidad por la } \\
\text { naturaloza. }\end{array}$ & $\begin{array}{l}\text { - So obtiene con la } \\
\text { aplicación del metodo } \\
\text { cientifico. }\end{array}$ & $\begin{array}{l}\text { - La Biologia se construye } \\
\text { solo a travós do procesos } \\
\text { de experimentacion. }\end{array}$ & $\begin{array}{l}\text { - Incorpora la visión } \\
\text { historica en el trabajo } \\
\text { conceptasal y la } \\
\text { apropiación del lenguaje } \\
\text { técnico de is disciplina. }\end{array}$ & $\begin{array}{l}\text { - So obtione a través ce la } \\
\text { experimentación y la } \\
\text { aplicacaión dol mótodo } \\
\text { científico. }\end{array}$ \\
\hline $\begin{array}{l}\text { 2. IMAGEN DE LA } \\
\text { PROFESION } \\
\text { DOCENTE } \\
\text { a. Dificultad }\end{array}$ & $\begin{array}{l}\text { - Ubicadias en los } \\
\text { ostudiantos (poreza, falta } \\
\text { de interes). }\end{array}$ & $\begin{array}{l}\text { - Poca consulta do los } \\
\text { estuciantes, pero no de } \\
\text { dificultades en los } \\
\text { aprendizajes. }\end{array}$ & $\begin{array}{l}\text { - Alumnos perezosos a } \\
\text { irresponsables. }\end{array}$ & - Manitiesta dificultad on el. & $\begin{array}{l}\text { - Accoso a textos de } \\
\text { consulta por parte de los } \\
\text { alumnos. }\end{array}$ & $\begin{array}{l}\text { - Reconoce dificultades } \\
\text { para ejercer como doconto. }\end{array}$ \\
\hline 0. ngrado & $\begin{array}{l}\text { - Familiaridad on el trato con } \\
\text { los jovenes aunque con } \\
\text { rigor. }\end{array}$ & $\begin{array}{l}\text { - Le interesa la interacción } \\
\text { en los estudiantes y } \\
\text { aprende de ellos. }\end{array}$ & $\begin{array}{l}\text { - Interacción con los } \\
\text { jóvenos. }\end{array}$ & - No se tiene evidencia. & $\begin{array}{l}\text { - Trabajo en grupo de } \\
\text { docentos y roconocorse } \\
\text { como constructores de } \\
\text { saber podagógico. }\end{array}$ & $\begin{array}{l}\text { - No maniliesta agrado por } \\
\text { la profesión docente. }\end{array}$ \\
\hline $\begin{array}{l}\text { c. Prolesión y opción def } \\
\text { vida }\end{array}$ & - Segunda opción. & $\begin{array}{l}\text { - Segunda opción, aunque } \\
\text { distruta el trabajo docente. }\end{array}$ & $\begin{array}{l}\text { - Segunda opción (medicina } \\
19 \text { ). }\end{array}$ & $\begin{array}{l}\text { - Tercora opción (medicina } \\
\left.\because \text { quimica } 2^{*}\right) \text {. }\end{array}$ & $\begin{array}{l}- \text { Tercera opción (Medicina } \\
1 \% \text {, Cuimica } 2^{\circ} \text { ). }\end{array}$ & $\begin{array}{l}\text { - Segunda opción } \\
\text { (Ingenioria quimica 12). }\end{array}$ \\
\hline
\end{tabular}


Esta coherencia, es posible verla desde dos perspectivas: una, que su actuar en el aula es armónico, respecto a sus intencionalidades, en la mayoría de los casos; y, dos, la relación pensamiento-acción, muestra articulación. Cabe aclarar, que para nosotros, el pensamiento del profesor no lo deducimos de sus expresiones sobre el deber ser de la educación - frente a lo cual los docentes expresan ideas bastante similares y relacionadas con el discurso que circula en las políticas educativas-, sino que el pensamiento o las ideas más potentes en las pautas de acción de los docentes. las evidenciamos a través del análisis de sus acciones cotidianas en el aula y en las razones expresadas durante la entrevista en profundidad, sobre las intencionalidades y razones de ciertas decisiones en el transcurrir cotidiano de las clases, momento en el cual se dejan ver las arraigadas convicciones que orientan el quehacer de los docentes y en las cuales basa su trabajo pedagógico.

Como lo señalan Fernández y Elortegui (1996), el trabajo como docente tiene un carácter eminentemente práctico, aunque detrás de ella debe haber un cuerpo teórico que explique en qué fundamento se basa. Ahora bien, en los distintos momentos interactivos con los docentes, se pudo evidenciar que tienen un saber sobre su práctica pedagógica, que hace falta sistematizar y racionalizar, al tiempo que hacerlo consciente. Esto se hizo posible a través de la realización de esta investigación, para los casos trabajados.

Cabe destacar, la excelente acogida de los docentes en torno a estas actividades, su colaboración y el reconocimiento unánime acerca de los logros que para ellos significaba la toma de conciencia de las fortalezas y limitaciones de su(s) propio(s) modelo de actuación y el compromiso en la búsqueda de alternativas para cualificar su trabajo en el aula, así como la formación en valores en los estudiantes: todo ello, teniendo en cuenta las características de cada contexto y de cada profesor. Es más, durante la socialización de los resultado, los docentes destacaban la importancia de reconocerse en cuanto a sus concepciones y sus acciones.

Ahora bien, esta toma de conciencia, mediante la participación del profesor en la investigación le posibilité la transformación de sus prácticas pedagógicas, lo cual contribuyó a la cualificación de la enseñanza de la Biología, uno de los propósitos de este proyecto.

Somos conscientes que la información registrada en éste documento y en los archivos de la investigación pueden dar lugar a otras descripciones e interpretaciones, según la perspectiva desde donde se aborden; por ejemplo, trabajar en profundidad cada creencia, detallar otros elementos de cada esquema de pensamiento, en búsqueda de nuevos significados y sentidos, es una tarea que se nos plantea como un campo abierto a futuras indagaciones, para consolidar esta línea de investigación en Colombia.

\section{Creencias y acciones de los profesores participantes en el estudio}

Teniendo en cuenta la interpretación conjunta elaborada entre maestros e investigadores, encontramos una serie de aspectos relevantes, que consideramos importante enunciar, respecto a las búsquedas que guiaban los propósitos planteados para este estudio. Muchos de los aspectos encontrados muestran las fortalezas, el dinamismo y creatividad que se expresa en las diversas formas como los maestros intentan y logran desarrollar lo que para ellos se constituye en los fines que guían sus acciones, otros aspectos se constituyen de por si, en referentes que pueden servir de base para profundizar su análisis o para orientar nuevas búsquedas, en futuros proyectos de investigación. Con el fin de ilustrar parcialmente algunas de las creencias que hacen parte del pensamiento de 
los docentes de este estudio, hemos introducido en el texto expresiones de los profesores que pueden evidenciarlas.

Las creencias que mostraremos a continuación se basan en planteamientos que pueden dificultar los procesos educativos en que están empeñados los maestros; por esta razón nos permitimos mostrarlas y analizarlas. (Sin ignorar que los docentes dejan ver una buena cantidad acciones que muestran la entrega y el compromiso con que los profesores asumen su trabajo y que ameritan ser emuladas; pueden ser identificadas según el criterio del lector, en el Cuadro 1).

\section{Estas creencias son:}

1. A pesar de que el profesor hoy cuenta con normas educativas que le posibilitan expresar su autonomía y también con discursos pedagógicos que le permiten ampliar su pensamiento educativo, aún sigue manifestando que las exigencias institucionales y sociales le obligan a centrar la enseñanza en los contenidos. Por tanto, los estudiantes no participan en la escogencia de los temas y éstos no surgen de los intereses de los chicos, aunque el profesor en algunos momentos deja abierta la posibilidad de preguntar y ampliar libremente las temáticas. Al respecto el profesor Benjamín manifiesta: "Sí me da mucho susto en décimo por la cuestión del ICFES. El programa es tan extenso. Para este docente es importante el cumplimiento del programa y aunque posibilita el trabajo participativo de los estudiantes, éste sólo se hace dentro de los limites de los temas seleccionados por el docente. Además, le parece difícil el trabajo por proyectos con los estudiantes: si, el que está en el manual del Ministerio de Educación donde está la unidad . . pues básicamente se siguen las unidades del programa... para mí es muy difícil usar el proyecto...

2. Suponer que relacionar lo aprendido con la cotidianidad, permite la construcción de conocimiento biológico; sin poner en cuestión que, en la mayoría de los casos, las construcciones científicas contradicen el saber cotidiano entendido desde el sentido común. para que él entienda esa situación uno se vale de ejemplos sencillos de cosas cotidianas... ¿ ¿se acuerdan cuando ustedes eran pequeñitos y tenían una cantidad de juguetes, ustedes qué hacian ${ }^{9}$, señala y pregunta Darío para introducir el tema de la clasificación.

3. Continúan con la idea de que el texto es el portador del saber, regulador de contenidos y conocimientos en la escuela, en contraposición con su queja del poco acceso a los textos, por parte de los alumnos. Si bien el profesor Benjamin no usa un único texto guía, si asume los textos como portadores de saber y como un referente teórico más: "...sí, un texto en especial no tengo...me ayudan mucho con ciertos talleres... hay lecturas interesantes hay datos que uno consigue en el texto y que de pronto no va a conseguir en otro lado... parece que lo más fácil era llevar textos guía de bachillerato, Pero a pesar de darle importancia a las ideas de los estudiantes, finalmente el profesor explica que el texto resume bien y claramente, los aspectos que suscitaron dudas e inquietudes en la clase: "... vayamos al texto para aclarar un poquito sobre esto... hay un párrafo cortito que dice de una forma muy esquemática en pocas palabras cómo funciona esto. Desde otra perspectiva y por su formado trabajo, el profesor Darío no considera necesario tener un texto guía, pero reconoce que buena parte del saber está en los textos; de ésta manera, el texto regula el conocimiento en la escuela, que éste profesor ilustra cuando dice: "... Definitivamente el chico va tener que buscar un libro, va a tener que preguntar, partir de ahí es cuando so forma la persona que observa... 
4. Los profesores aunque consideran el acto de enseñar como complejo, en ellos mismos no ven mayores dificultades para su desempeño como docentes. El profesor Adolfo nos dice: ..."Se me ocurren las cosas en el momento y así no las tenga planeadas, yo cambio las cosas en ese momento..." . El profesor así, manifiesta su protagonismo en el aula, él es quien decide, haciéndose evidente su poder en el aula. La modificación de las actividades obedece a externalidades donde las circunstancias, lo condicionan a variar lo planeado: "...dependiendo del grupo y de la clase uno siempre hace cosas que le sugiere el momento o que en el momento se le ocurren por las circunstancias". Mientras que para el profesor Benjamín, la actitud y el sentido que el docente muestre por la materia a enseñar, estimula el interés del estudiante "...Sí, yo me doy cuenta que cuando a mí me interesa mucho el tema, ellos se interesan; en cambio a veces, digamos cuando yo no le he encontrado todo el sentido a un tema, pues ellos tampoco; o sea, es una situación tan sutil como que de pronto la actitud del maestro... yo me he dado cuenta que cuando llego con ánimo, con energía, con ganas, ellos trabajan más motivados"... Para el profesor Ernesto además "...el chico se pasa jugando con pepitas y se le va quedando"... Acá nos preguntamos ¿qué pasa entre el jugar y el aprender?, ¿a través de qué procesos se lograría la apropiación o construcción de teorías, o qué pasa con la conceptualización en los estudiantes? Ello no ofrece dificultades?

Pocos profesores como Benjamín consideran la enseñanza una actividad difícil, reconociendo sus dificultades. "... he tenido muchos conflictos sobre todo conflictos conmigo. A ver, la dificultad básica que es, ser demasiado teórico..”, aunque no deja de quejarse por la poca consulta de los estudiantes. El profesor Francisco, reconoce: las personas a veces ven eso que el trabajo del maestro, que es muy rico, que es muy fácil pero en realidad no es que sea muy fácil ; especialmente las externas a él la misma infraestructura del colegio no se presta para muchas cosas....

5. En general, los profesores mantienen la concepción que las metodologías exitosas en la enseñanza son las relacionadas con lo instrumental, lo práctico, las salidas, la experiencia directa de los alumnos, o mediante la aplicación del método científico desde una perspectiva empirista y poco tienen en cuenta trabajar en la lógica del pensamiento del niño y del joven, para entendería y de ser el caso transformarla. Es el caso del profesor César: "en el laboratorio la ventaja es que el estudiante está todo el tiempo partiendo de la observación y llega a una conclusión, entonces el método científico se está practicando. Acá se hace evidente, además, un reduccionismo del método científico" a la sola observación y conclusión y una ilusión de cientificidad por el intento de aplicación de algunas de sus orientaciones; este docente no toma en cuenta por ejemplo, la importancia de la pregunta que guíe las búsquedas e indagaciones, tampoco aparecen los referentes anteriores que puedan servir de base a la formulación de hipótesis, no hay cabida a un planteamiento divergente ni mucho menos a la argumentación que está en la base del pensamiento racional.

6. La mayoría de los profesores considera que el aprendizaje en los alumnos depende de la acción del profesor y éste no percibe que los estudiantes puedan tener dificultades en el aprendizaje de los conceptos. Para el profesor Francisco el aprendizaje depende de él ..."porque yo les desperté ese interés, yo trabajé con ellos en clase... los alumnos se interesan por mí, por mi clase... si, es que a veces planea una cosa y no le sale, entonces le toca pensar otra estrategia...".

7. La mayoría de los profesores del grupo de estudio, considera que la Biología es una mezcla de disciplinas; para el profesor Darío: “...el chico debe tener primero de pronto 
una formación matemática para poder entender la parte de biología y de química, por ejemplo, uno le habla de volumen y no entiende que es volumen, entonces uno tiene que empezar por enseñarle todas esas cositas...." Según ellos, dada la obviedad de los objetos biológicos, ésta es fácil de aprender, y para Adolfo, "...Porque esas cosas son más fáciles de tratar. O sea, a el muchacho las relaciona más con su vida cotidiana le queda más fácil entenderlas...". El profesor desconoce el carácter abstracto de la Biología como discurso sobre los seres de la naturaleza.

8. Dada la incertidumbre que introducen las recientes innovaciones educativas, el profesor no ha construido un imaginario acerca de la evaluación, mas allá de pensarla como una mera valoración de contenidos y actitudes según su singular forma de verlos. El profesor Darío utiliza variedad de instrumentos y maneras de evaluar; pareciera que el interés está en la promoción: "Por favor me le anotan la participación... ustedes ya saben cómo es, se les va acumulando puntaje y se les tiene en cuenta al finalizar el trimestre para efectos de la nota...”.

9. Si bien hay en los profesores un profundo respeto y valoración por la carrera docente, en la mayoría de los casos esta profesión, fue para ellos una segunda o tercera opción en sus proyectos de vida. Adolfo nos expresa que la profesión docente fue una segunda opción en su vida y no quería ser maestro, pues su deseo siempre fue ser un gran investigador "yo nunca quería ser maestro... mi deseo toda mi vida fue como vincularme a algo de investigación, así como en laboratorios..." . Esto se refleja en la acción en el aula cuando el docente hace éntasis en el trabajo de laboratorio. Sin embargo, el profesor reconoce que su imagen acerca de la profesión docente ha cambiado: Para mí ser maestro era algo... la persona que está ocupada en... de una educación y con muchísima responsabilidad... pero cuando yo estuve, ya en el contacto con los niños y comencé a ver los resultados de lo que yo hacia... entonces esas cosas me alentaron mucho que me llevaron a amar el ser maestro".

Finalmente, el proceso de toma de conciencia y transformación de las prácticas de enseñanza de los docentes se favorece con los espacios de participación en procesos de investigación sobre su propia acción y dentro del propio contexto, a través del trabajo en equipo con los investigadores.

\section{CONSIDERACIONES FINALES}

Además del análisis de todas las implicaciones de las anteriores creencias en la acción del profesor, el desarrollo de la presente investigación nos puso en contacto con una serie de problemáticas que por su naturaleza se apartaban un poco de los propósitos que eran objeto de este estudio pero que consideramos oportuno mencionar, con el ánimo de empezar a abrir espacios de toma de conciencia y de debate hacia la búsqueda de alternativas y el desarrollo de nuevos trabajos en la Línea del Pensamiento del Profesor.

- Es importante tener en cuenta que aunque al profesor, no se le preparó para asumir las exigencias educativas actuales y enfrentar los nuevos retos, cabe destacar los esfuerzos de grupos de maestros y de las redes de cualificación y grupos de investigación, que han permitido ir abordando de manera reflexiva y crítica el quehacer en la escuela.

- Por el énfasis en los enfoques ambientalistas y algunos cuestionamientos a los métodos memorísticos en la enseñanza; así como, la invasión de modelos didácticos 
innovativos, se ha puesto al profesor en encrucijada. Esto ha traído como consecuencia, que los docentes empiezan a abandonar el discurso propio de la biología, al cual habría que prestarle mucha atención, para no perder las perspectivas de un saber hoy tan necesario para la humanidad.

- Muy sorprendente resulta, que a pesar de la abundante y buena información que hoy existe acerca del aprendizaje; el profesor no tiene en cuenta que el estudiante pueda tener dificultades de aprendizaje. Pareciera que hay una excesiva confianza, en que el estudiante por si solo puede lograr construir todos los conocimientos, ¿secuelas de un modelo constructivista mal entendido? Es así como, muchos profesores no orientan de manera conveniente la construcción o elaboración de conceptos. Todo ello hace crisis, cuando el profesor enfrenta los nuevos modelos evaluativos.

- Es evidente que las creencias que estuvimos trabajando en secundaria, pueden tener otro tipo de manifestaciones en niveles distintos del sistema educativo

\section{AGRADECIMIENTOS}

El grupo de investigación desea expresar sus agradecimientos a:

Los profesores de Ciencias Naturales y Educación Ambiental, que permitieron el acceso del equipo de investigación a sus clases, posibilitaron todos los espacios de intercambio de ideas y experiencias y se prestaron para auscultar aspectos de la especificidad de su pensamiento educativo superando sus temores, y, abiertos siempre a la crítica y a la construcción de alternativas para mejorar su trabajo.

A la Universidad Pedagógica Nacional, Departamento de Biología y División de Gestión de Proyectos CIUP, por el apoyo financiero y logístico.

\section{BIBLIOGRAFÍA}

Barquín, J. (1987). "La investigación sobre el profesorado. Estado de la cuestión en España”. En: Revista de Educación. No. 306. Pp.7-65.

ChonaG., Castaño N., Arteta J., Leudo M., Valencia S. y Martínez S. (1998). "Aproximación a las creencias que orientan la práctica del profesor de Biología". Revista TED de la Facultadde Ciencia y Tecnología. Universidad Pedagógica Nacional. No. 4. Pp. 93-104.

Dewey J. (1989). Cómo pensamos. Nueva exposición de la relación entre pensamiento y proceso educativo. Barcelona: Paidós.

Fernández, J., Elortegui, N. (1996). Qué piensan los profesores acerca de cómo se debe enseñar. Enseñanza de las ciencias, 14(3). Pp. 331-342.

Furió, C. (1994). Tendencias actuales en la formación del profesorado de ciencias. Enseñanza de las Ciencias, 12(2). Pp.1 88-199. 
Gallego, M. (1991). Investigación sobre pensamientos del profesor: Aproximaciones al estudio de las teorías y creencias de los profesores. Rey. Española de Pedagogía No. 189. Pp. 287-325.

Giordan, A. (1982). La enseñanza de las ciencias. Editorial Siglo XXI. Madrid.

Gagliardi, A. (1986). Interés didáctico de los errores de los alumnos. Enseñanza de las Ciencias, 3(1). Pp. 11-17.

Marcelo C. (1987), citado en Gallego, M. 1991. Investigación sobro pensamientos del profesor: Aproximaciones al estudio de las teorías y creencias de los profesores. Rey. Española de Pedagogía No. 189. Pp.287-325.

Mellado J. (1996). "Concepciones y prácticas de aula de profesores de ciencias". En: Formación inicial de primaría y secundaria. Revista Enseñanza dalas Ciencias. 14(2). Pp. 289-302.

Munby, H. (1982). "Investigación sobre el pensamiento deles profesores: Dilemas ante la conducta y práctica profesionales”. En: Villar L. (1888). Conocimiento, Creencias y Teorías de los Profesores. Editorial Marfil. España.

Novack, J. y Gowin, B.(1988). Aprendiendo a Aprender. Martínez-Roca. Barcelona.

Pacca, J. : Gircoreano, J. ; Henrique, K. F. Higa, I. y Sbruzzi L. (1997). Las actividades de aula y la conducta del profesor. Enseñanza de las ciencias. Número Extra. Pp. 63-64.

Pérez A. y Gimeno J. (1988). Citado en Gallego, M. 1991. Investigación sobre pensamientos del profesor: Aproximaciones al estudio de las teorías y creencias de los profesores. Rev. Española de Pedagogía No. 189. Pp. 287-325.

Parlón R., Rivero A. y Martin del Pozo, R. (1997). Conocimiento profesional y epistemología de los profesores l: Teoría, métodos e instrumentes. Enseñanza de las Ciencias 15 (2). Pp. 155-164.

Reyes L. y Salcedo L. (1998). Acciones de maestres de ciencias: Creencias, roles, metas y con textos en la enseñanza y el aprendizaje. Revista TED de la Facultad de Ciencia y Tecnología No. 3.Pp. 5-17.

Reyes L., Salcedo; L., Perafán, G. (1999). Acciones y creencias, Tesoro oculto del educador. Tomo 1. Arfo. Bogotá.

Yerrit R., Parke L. Y Nugent J. (1997) Citado en: Reyes L., Salcedo L., Perafan G (1999). Acciones y Creencias, Tesoro Oculto del Educador. Tomo I.. Arfo. Bogotá. 


\section{ANEXO No. 1 \\ CREENCIAS A LAS CUALES HACEMOS SEGUIMIENTO (Ver Chona et al. 1998)}

A partir de las razones dadas por los profesores de Biología es posible entrever que sus creencias están caracterizadas por consideraciones coma las siguientes:

- La biología es una mezcla de disciplinas, tiende a ser empírica y especulativa porque siempre da respuestas en términos de aplicación. Tiene implicaciones políticas, sociales y económicas especialmente relacionadas con la ecología, la salud, la conservación y biodiversidad y el mejoramiento de la vivencia humana.

- La experimentación biológica se centra en acciones educativas relacionadas con la aplicación del método científico. Las prácticas de laboratorio generan espíritu científico.

- Se pierde la esencia de la biología si la enseñanza toma en consideración los intereses de los estudiantes.

- Las exigencias institucionales y sociales obligan a centrar la enseñanza en los contenidos y cuando el profesar trata de pensar en estos, los alumnos no son tan relevantes.

- Las metodologías exitosas en la enseñanza están relacionadas con lo instrumental, la práctica, las salidas, la experiencia directa de los estudiantes.

- El texto es portador de saber y regulador de los contenidos y del conocimiento en la escuela.

- La enseñanza de las ciencias depende fundamentalmente del maestro, especialmente de su metodología, habilidad y creatividad.

- El aprendizaje de las ciencias depende de la acción del profesor y de la actividad y participación del estudiante.

- En la enseñanza de la biología las dificultades se limitan a la falta de textos específicas y a la poca consulta por parte de los estudiantes.

- La ciencia más difícil de aprender es la física, seguida de la química, por el nivel de abstracción que requieren. 